\title{
Amlodipine and valsartan as components of a rational and effective fixed-dose combination
}

\author{
Bernard Waeber' \\ Luis M Ruilope ${ }^{2}$ \\ 'Division of Clinical Pathophysiology, \\ University Hospital, Faculty of Biology \\ and Medicine, University of Lausanne, \\ Switzerland; ${ }^{2}$ Hypertension Unit, \\ Hospital 12 de Octubre, Madrid, Spain
}

Correspondence: CHUV, Prof. Bernard Waeber

Division of Clinical Pathophysiology, PPA MP I4/204, CH-I0I I Lausanne, Switzerland

Tel $+4 \mid$ I 2 I 3140760

Fax +4l 2I 3|4 25।8

Email bernard.waeber@chuv.ch

\begin{abstract}
Pharmacological treatment of hypertension is effective in preventing cardiovascular and renal complications. Calcium antagonists and blockers of the renin-angiotensin system are widely used today to initiate antihypertensive therapy but, when given as monotherapy, do not suffice in most patients to normalize blood pressure. Combining the two types of agents considerably increases the antihypertensive efficacy, but not at the expense of a deterioration of tolerability. This is exemplified by the experience accumulated with the recently developed fixed dose combination containing the $\mathrm{AT}_{1}$-receptor blocker valsartan $(160 \mathrm{mg})$ and the dihydropyridine amlodipine (5 or $10 \mathrm{mg}$ ). In a randomized trial, an 8-week treatment normalized blood pressure $(<140 / 90 \mathrm{mmHg})$ within 8 weeks in a large fraction of hypertensive patients (78.4\% and $85.2 \%$ using the $5 / 160[\mathrm{n}=371]$ and $10 / 160 \mathrm{mg}[\mathrm{n}=377]$ dosage, respectively). Like all $\mathrm{AT}_{1}$-receptor blockers valsartan has a placebo-like tolerability. Valsartan prevents to a large extent the occurrence amlodipine-induced peripheral edema. Both amlodipine and valsartan have beneficial effects on cardiovascular morbidity and mortality, as well as protective effects on renal function. The co-administration of these two agents is therefore very attractive, as it enables a rapid and sustained blood pressure control in hypertensive patients. The availability of a fixed-dose combination based on amlodipine and valsartan is expected therefore to facilitate the management of hypertension, to improve long-term adherence with antihypertensive therapy and, ultimately, to have a positive impact on cardiovascular and renal outcomes.
\end{abstract}

Keywords: antihypertensive therapy, fixed-dose combination, calcium antagonists, angiotensin receptor blockers, blood pressure control, tolerability

\section{Introduction}

Hypertension has long been recognized as a major independent risk factor for cardiovascular and renal diseases and still represents worldwide a leading cause of mortality, both in industrialized and developing countries. ${ }^{1,2}$ Pharmacological treatment of hypertension is very effective in protecting against complications such as stroke, myocardial infarction, heart failure and deterioration of renal function. ${ }^{3-5}$ Calcium antagonists (CAs) and blockers of the renin-angiotensin system, in particular angiotensin converting enzyme inhibitors (ACE-Is) and angiotensin II receptor blockers (ARBs), are widely used antihypertensive drugs with well documented beneficial effects, as shown repeatedly in individual trials and meta-analysis..$^{3-5}$ This led international experts to consider these classes of blood pressurelowering agents as valuable options to initiate antihypertensive therapy. ${ }^{6}$ It is recognized, however, that any antihypertensive drug, regardless of its mechanism of action, allows blood pressure normalization in only a fraction of patients, so that combination therapy is required in most patients to achieve strict blood pressure control. ${ }^{6,7}$ The co-administration in a single tablet of 2 drugs acting on different pressor systems permits at the same time to improve the blood pressure control rate and to simplify the treatment, thereby facilitating the long-term adherence with therapy. ${ }^{8,9}$ No wonder therefore that the interest for fixeddose combinations in the field of hypertension is growing rapidly. 
Amlodipine (dihydropyridine CA) and valsartan (ARB) have been evaluated in numerous interventional trials and have become very popular in the management of hypertensive patients. ${ }^{3-5} \mathrm{~A}$ fixed-dose combination containing amlodipine and valsartan has been recently developed. ${ }^{10}$ The present paper reviews the rationale for co-administering these two agents, drawing on the experience accumulated so far with this new drug combination.

\section{Amlodipine and valsartan: two antihypertensive agents with different mechanisms of action}

CAs have been extensively studied during the last 3 decades and have emerged as a major advance in the management of diverse cardiovascular diseases, including hypertension. CAs represent a heterogeneous group of agents. ${ }^{11,12}$ They all block the transmembrane calcium influx in vascular and myocardial cells through L-type channels, being more or less selective for the vasculature and the heart. Dihydropyridines have the most potent vasodilatory effects, with no overt cardiac effects in humans. Amlodipine is a dihydropyridine characterized by a slow onset and a long duration: ${ }^{13}$ maximal plasma concentrations are not achieved until about 6 hours after dosing and the elimination half-life exceeds 35 hours. The vasodilation induced by calcium entry blockade may trigger a reflex increase in sympathetic nerve activity, possibly manifesting as a heart rate acceleration. ${ }^{14}$ If present, the hyperadrenergic state system might augment myocardial oxygen demand and be harmful in patients with coronary heart disease.${ }^{15}$ Notably, no evidence for a sustained activation of the sympathetic drive has been found in hypertensive patients treated with long-acting dihydropyridines, amlodipine included. ${ }^{16}$

Valsartan, like all ARBs, acts by inhibiting the binding of angiotensin II to the $\mathrm{AT}_{1}$-subtype of angiotensin II receptor. ${ }^{17,18}$ A hallmark of blockers of the renin-angiotensin system is their ability to lower blood pressure without usually inducing a reflex increase in sympathetic nerve activity, which is reflected by the absence of consistent changes in heart rate..$^{19,20}$ After oral administration, the peak plasma concentration of valsartan is reached after 3 hours, and the elimination half-life is 6 hours. This compound has already been investigated in a hypertension outcome trial. ${ }^{21}$

The antihypertensive efficacy of CAs and ARBs is dosedependent. For tolerability, however, a clear-cut dose-dependency exists only for CAs. ${ }^{8}$ The commonest symptoms of CAs, ie, flushing, ankle edema, headaches and palpitations, are caused by drug-induced vasodilation. ${ }^{14}$ The side-effects attributable to vasodilation are caused mainly by dihydropyridine derivatives. Headache, palpitations and flushing occur most often during initiation of treatment, and are reduced in frequency and/or severity by compounds such as amlodipine exhibiting a slow onset of action and smooth peak versus trough blood levels, thus avoiding excessive blood pressure fluctuations during the day. Ankle edema encountered during calcium entry blockade is not due to renal fluid retention but involves a rise in intracapillary pressure (as a consequence of a selective diminution of the precapillary arteriolar tone), with an ensuing leak of fluid into the interstitium. ${ }^{22}$ Valsartan, like all ARBs, exhibits a placebo-like tolerability. ${ }^{8,17}$

\section{Position of calcium antagonists and angiotensin receptor blockers in the treatment of hypertension}

To date, all currently available long-acting CAs and ARBs are considered by many national and international authorities as a valuable first-line option for the management of hypertension, for instance experts who prepared the most recent hypertension guidelines on behalf of the European Society of Hypertension (ESH) and the European Society of Cardiology (ESC). ${ }^{6}$ CAs and ARBs are generally equally effective in lowering blood pressure when compared in groups of unselected patients with mild to moderate hypertension. A given patient may, however, respond favorably to a CA, but not to an ARB, and vice versa. According to experts in UK, the age and the ethnicity may be helpful in guiding the choice of initial therapy: ${ }^{23}$ older patients and black patients of any age should receive preferably a CA, whereas younger patients are more likely to normalize their blood pressure using a blocker of the renin-angiotensin system, as renin secretion tends to decrease with aging. The magnitude of the blood pressure fall induced by antihypertensive drugs increases with the degree of hypertension, particularly when using vasodilators such as CAs.

There is ample evidence today that dihydropyridine CAs and ARBs have a positive impact on the cardiovascular, cerebrovascular and renal outcomes of hypertensive patients. ${ }^{3-5}$ This is especially true in high risk patients with multiple cardiovascular risk factors, subclinical target organ damage, or established cardiovascular or renal disease. Amlodipine and valsartan were part of the drug regimens under study in several large hypertension morbiditymortality trials, and much of the present knowledge on the beneficial effects of CAs and ARBs in various clinical conditions associated with high blood pressure has been 
accumulated using these two compounds. It is beyond the scope of this paper to review the evidence supporting the use of dihydropyridine CAs versus ARBs in the individual patient. Figure 1 summarizes only the principal indications of these agents as initial therapy, based on the most recent ESH/ESC recommendations. ${ }^{6}$ It appears that there are good reasons for using either a dihydropyridine or an ARB in the prevention and/or the treatment of most complications of hypertension. CAs antagonists and ARBs, including amlodipine and valsartan, are easy to use and can be administered to nearly all hypertensive patients, which represents a major advantage in everyday practice. Notably, long-acting dihydropyridines have no compelling contra-indication. ARBs should certainly not be given in pregnant women. They should be avoided or used only with close monitoring of renal function in patients with bilateral renal artery stenosis or a renal artery stenosis on a single kidney. In patients with unilateral renal artery stenosis, a drug regimen comprising a blocker of the renin-angiotensin system might, however, represent a valuable alternative to renal revascularization. ${ }^{24}$ ARBs tend to increase plasma potassium levels and should not be used in patients with hyperkaliemia, and not be coadministered with a potassium-sparing diuretic unless renal function is normal.

\section{Rationale for combining amlodipine and valsartan}

Dihydopyridines and ARBs have a major advantage: they can be administered safely with other classes of antihypertensive drugs, including with each other. Combining 2 agents lowering blood pressure by different mechanisms allows a major gain in antihypertensive efficacy compared with monotherapies, but usually not at the expense of a deterioration of tolerability. ${ }^{8}$ Notably, the counter-regulatory response of the

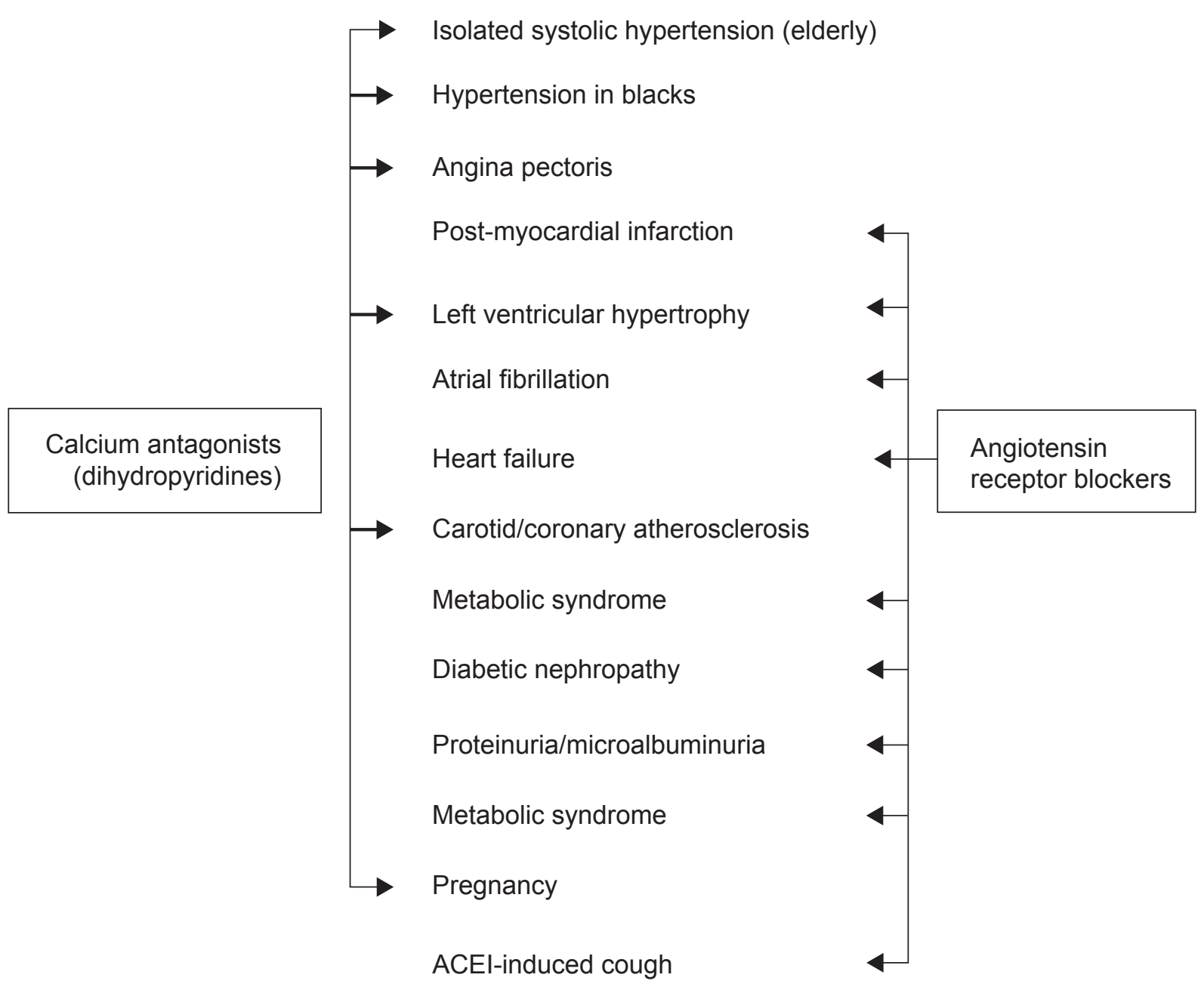

Figure I Conditions (indicated by arrows) favoring the use of dihydropyridine calcium antagonists and angiotensin receptor blockers according to the 2007 European Society of Hypertension/European Society of Hypertension Hypertension Recommendations. Adapted with permission from Mancia et al. J Hypertens. 2007;25: I I05-I I87. ${ }^{2}$ Copyright (C) 2007 Lippincott Williams \&Wilkins. 
sympathetic nervous system to the vasodilation induced by dihydropyridines is buffered by concomitant blockade of the renin-angiotensin system, which may contribute to enhancement of the blood pressure response as well as to prevention of the reflex increase in heart rate and the palpitations possibly occurring during calcium entry blockade..$^{25}$ Also of note is that dihydropyridines, unlike selective arterial vasodilators, have a natriuretic effect, which is expected to render blood pressure maintenance more angiotensin II-dependent. ${ }^{26}$

Much experience has been accumulated with amlodipine and valsartan, both in clinical trials and in routine conditions of use, as the two drugs are now distributed worldwide. ${ }^{3-5}$ It was therefore appealing to have available a fixed-dose combination containing these two agents with well-documented salutary effects. ${ }^{10}$ The appropriate doses of the two components were established in 2 multicenter, 8-week, randomized, double-blind, placebo-controlled, trials. ${ }^{27}$ Both studies included a 2-week washout period, which was followed by a single-blind, placebo run-in period of 2 to 4 weeks, and thereafter by a 8-week, double-blind, active-treatment period (Study 1:amlodipine in daily doses of 2.5 to $5 \mathrm{mg}$, valsartan in daily doses of 40,80, 160 or $320 \mathrm{mg}$, alone or in combination, or placebo; Study 2: amlodipine in a daily dose of $10 \mathrm{mg}$, valsartan in daily doses of 160 or $320 \mathrm{mg}$, alone or in combination, or placebo). There were overall significantly greater blood pressure reductions with the combination regimens compared with the individual components, and a positive dose-response was observed for all combinations. Table 1 depicts the results obtained in the 2478 studied patients. There was a clear-cut dose-dependent blood pressure lowering effect of both monotherapies, and the co-administration of the two compounds was significantly more effective, whatever the dosages of the two combined agents, than equivalent doses of monotherapies. The analysis of the total safety population

Table I Mean blood pressure reductions $(\mathrm{mmHg})$ induced by an 8-week once-daily, treatment with different doses of amlodipine and valsartan given alone or in combination.

\begin{tabular}{|c|c|c|c|c|}
\hline \multirow{7}{*}{ 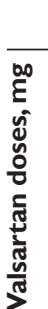 } & & \multicolumn{3}{|c|}{ Amlodipine doses, $\mathrm{mg}$} \\
\hline & & 0 & 2.5 & 5 \\
\hline & 0 & 7.3/7.1 & 12.4/9.3 & $15.1 / \mid 1.5$ \\
\hline & 40 & $11.8 / 10.1$ & $\mid 5.5^{* \# / /} 0.8$ & $19.6 * \# / 14.6 * \#$ \\
\hline & 80 & $12.9 / 9.7$ & $17.0^{* \# / / 3.4^{* \#}}$ & $20.8^{* \# / / 4.5^{* \#}}$ \\
\hline & 160 & $15.1 / \mid 1.0$ & $16.7^{\# / / 3.3^{* \#}}$ & $19.5^{* \# / / 4.2 * \#}$ \\
\hline & 320 & $15.7 / 13.4$ & $18.3^{\# /} / 14.2^{* \#}$ & $22.7^{* \# / / 5.9 * \#}$ \\
\hline
\end{tabular}

${ }^{*} \mathrm{p}<0.05$ versus the same dose of valsartan monotherapy.

$\# \mathrm{p}<0.05$ versus the same dose of amlodipine monotherapy.

Adapted with permission from Philipp et al. Clin Ther. 2007;29:563-580. ${ }^{27}$ Copyright (C) Elsevier.
( $n=3155)$ showed no significant difference in the overall rate of adverse events between patients treated with amlodipine - valsartan (44.1\%) and those having received amlodipine alone $(45.7 \%)$, but was greater $(\mathrm{p}<0.05)$ in patients on the combination than in those on valsartan monotherapy (39.8\%). The incidence of peripheral edema was lower $(\mathrm{p}<0.05)$ with amlodipine and valsartan given in combination (5.4\%) than with amlodipine alone $(8.7 \%)$, but higher $(\mathrm{p}<0.05)$ compared with valsartan monotherapy (2.1\%). The rate of adverse events leading to discontinuation of the treatment was low in the amlodipine-valsartan groups (1.8\%) and was comparable to that of placebo (2.1\%). Headache was the second most frequent adverse event after peripheral edema. It was encountered less frequently $(\mathrm{p}<0.05)$ in patients on amlodipine-valsartan combination (4.3\%) than in those administered amlodipine monotherapy (7.6\%).

It is worth to discussing here in more detail the development of peripheral edema, a common and troublesome adverse effect of CAs. ${ }^{22}$ As already mentioned, the fluid retention occurring during treatment with these agents involves a rise in intracapillary pressure, as a consequence of a selective diminution of the precapillar tone, with an ensuing net leak of fluid into the interstitium. Blockers of the renin-angiotensin system attenuate this process, most likely because of their ability to dilate not only the arteriolar vascular bed, but also the venous capacitance vessels. This was proven by a study in which the ankle foot volume was assessed by the principle of water displacement in 130 hypertensive patients randomized to receive for 6 weeks, according a cross-over design, either valsartan $160 \mathrm{mg}$ /day, amlodipine $10 \mathrm{mg} /$ day, or a combination of the two. ${ }^{28}$ Amlodipine, but not valsartan, significantly increased ankle foot volume, the latter being, however, significantly smaller during the coadministration of amlodipine and valsartan compared with amlodipine monotherapy.

\section{Antihypertensive efficacy and safety of amlodipine and valsartan as mono- or combination therapy in stage 2 hypertension and elderly hypertensive patients}

As pointed out above, the co-administration of amlodipine and valsartan is more effective in lowering blood pressure than the corresponding monotherapies. There is a need for such an increased efficacy especially in patients with blood pressure levels largely above the normal upper values, for example in those with stage 2 hypertension 
(systolic blood pressure $\geq 160 \mathrm{mmHg}$ and/or diastolic blood pressure $\geq 100 \mathrm{mmHg}$ ). Actually, the use of combination therapy is most often required in such patients to normalize blood pressure, so that fixed dose combinations are considered a valuable choice to initiate therapy in this subgroup of patients. ${ }^{6}$ The efficacy of amlodipine and valsartan, alone as well as in combination, has been compared in patients with type 2 hypertension and in patients with type 1 hypertension (systolic blood pressure of 140 to $159 \mathrm{mmHg}$ and/or diastolic blood pressure of 90 to $99 \mathrm{mmHg}){ }^{29}$ This was done by performing a post-hoc subgroup analysis of the two double-blind, randomized, placebo-controlled trials, that were similarly designed as already described above. ${ }^{27}$ A total of 1355 and 1800 patients had stage 1 and stage 2 hypertension, respectively. Baseline blood pressure averaged $145.9 / 96.8 \mathrm{mmHg}$ in the former, and 160.8/101.0 $\mathrm{mmHg}$ in the latter. In both trials, patients with stage 2 hypertension experienced greater reductions in systolic blood pressure than those with stage 1 hypertension. The maximum effects were obtained with the higher dose combinations in the two subgroups of patients. Figure 2 illustrates the changes in blood pressure in response to the $5 \mathrm{mg}$ dose of amlodipine
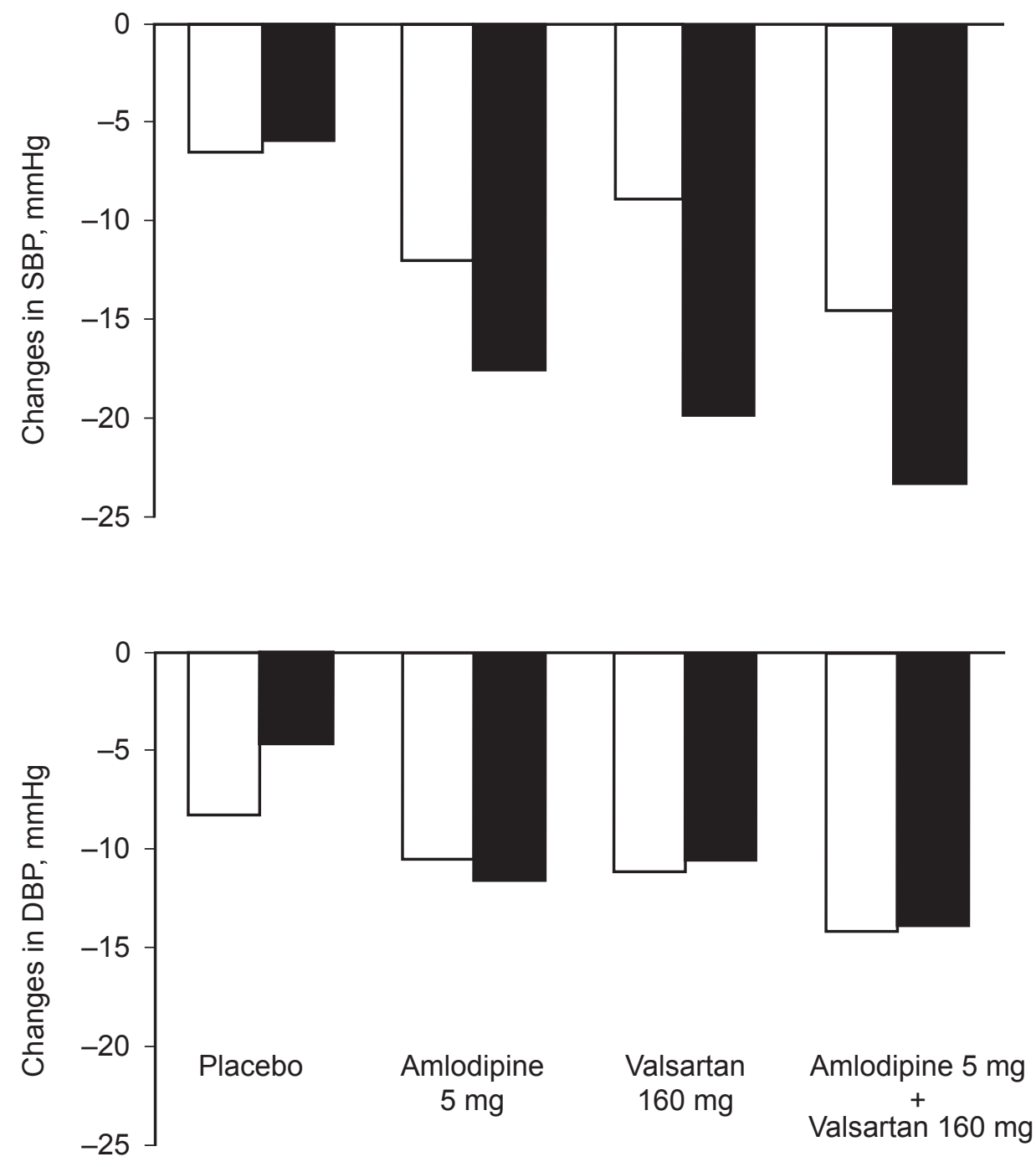

Stage 1 Hypertension

Stage 2 Hypertension

Figure 2 Changes in systolic (SBP) and diastolic (DBP) blood pressure induced by an 8-week treatment with amlodipine $5 \mathrm{mg}$ and valsartan $160 \mathrm{mg}$, alone or in combination, compared with placebo, in patients with stage I and stage 2 hypertension. Adapted with permission from Smith et al.J Clin Hypertens (Greenwich). 2007;9:355-364. ${ }^{29}$ Copyright (C) 2007 John Wiley \& Sons, Inc. 
and $160 \mathrm{mg}$ dose of valsartan, as mono- or combination therapy. At these doses, the two agents lowered blood pressure by an average of 14.6/14.2 $\mathrm{mmHg}$ in stage 1 hypertension, compared with $23.2 / 13.9 \mathrm{mmHg}$ in stage 2 hypertension (Study 1). The corresponding blood pressure reductions induced by combining $10 \mathrm{mg}$ of amlodipine and $160 \mathrm{mg}$ of valsartan averaged 20.3/16.5 $\mathrm{mmHg}$ and 29.6/17.6 $\mathrm{mmHg}$, respectively (Study 2 ). The lowest doses commonly combined in everyday practice (amlodipine $5 \mathrm{mg}$ and valsartan $80 \mathrm{mg}$ ) were also more effective in stage $2(-24.2 /-14.6 \mathrm{mmHg})$ than in stage 1 hypertension $(-16.9 /-13.9 \mathrm{mmHg})$.

Another issue is the influence of age on the blood pressure response to amlodipine and valsartan, alone or in combination. The patients enrolled in the two controlled trials reported by Smith and colleagues were divided in two subgroups, ie, the older (65 years or older; $n=704$; baseline blood pressure $=160.2 / 98.6 \mathrm{mmHg}$ ) ) and the younger (less than 65 years; $n=2451$; baseline blood pressure $=152.7 / 99.4 \mathrm{mmHg}$ ) patients. ${ }^{29}$ All active therapies produced greater blood pressure reductions than placebo. Figure 3 depicts the blood pressure changes induced by the $5 \mathrm{mg}$ amlodipine dose and $160 \mathrm{mg}$ of valsartan dose, alone or in combination. Notably, both agents given alone were more effective in lowering blood pressure in older than in younger subjects. In the older patients, the response of systolic blood pressure to amlodipine was greater than the corresponding response to valsartan. The co-administration of the two agents decreased blood pressure to a similar
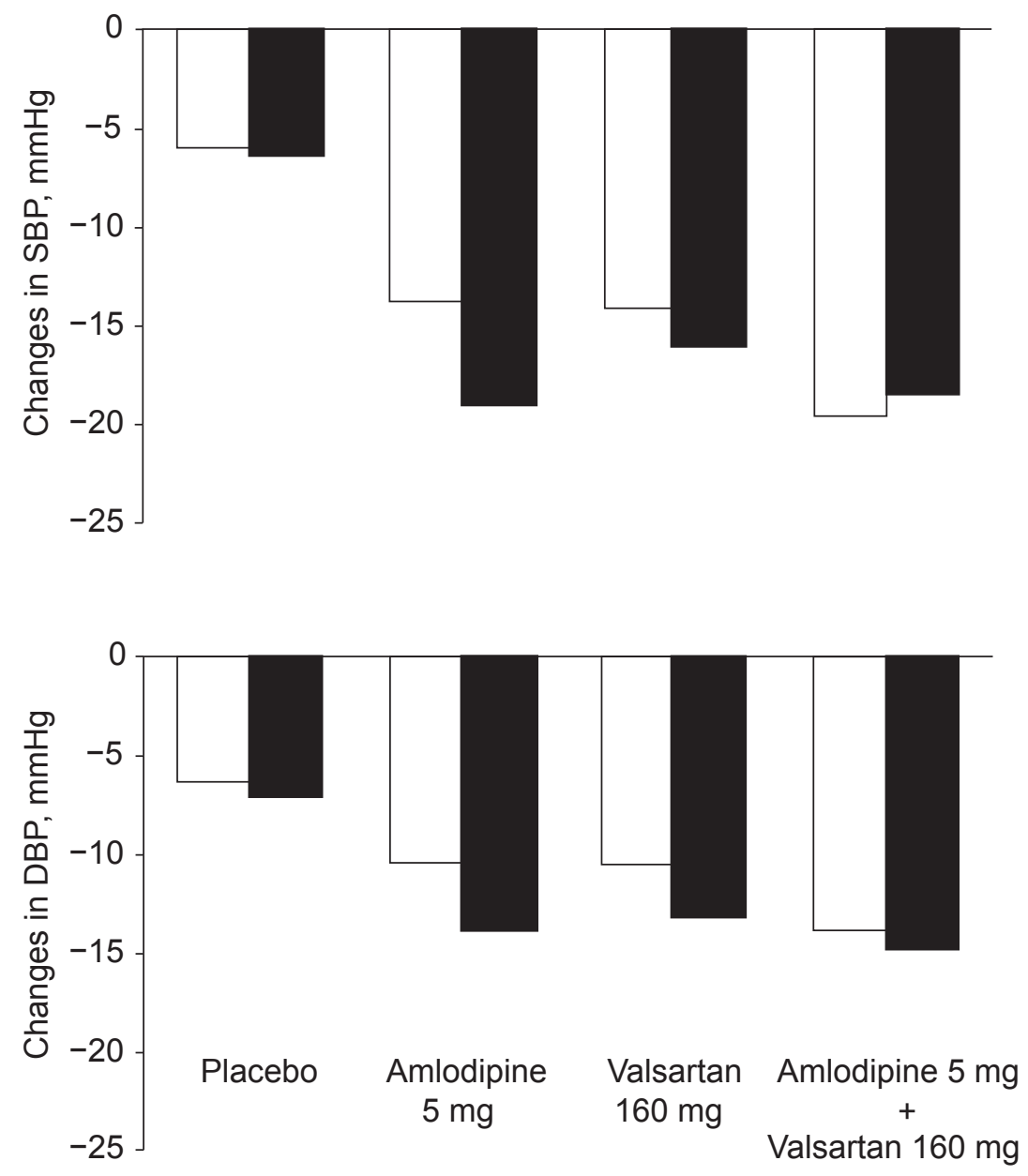

$<65$ years

$\geq 65$ years

Figure 3 Changes in systolic (SBP) and diastolic (DBP) blood pressure induced by an 8-week treatment with amlodipine 5 mg and valsartan I60 mg, alone or in combination, compared with placebo, in older and younger patients. Adapted with permission from Smith et al.J Clin Hypertens (Greenwich). 2007;9:355-364. ${ }^{29}$ Copyright @ 2007 John Wiley \& Sons, Inc. 
magnitude in the two age groups. Using the amlodipine $10 \mathrm{mg}$ valsartan/160 mg combination, blood pressure was reduced by $25.2 / 15.7 \mathrm{mmHg}$ and $27.0 / 17.8 \mathrm{mmHg}$ in the older and younger patients, respectively.

Ethnicity is another factor having the potential to influence the antihypertensive efficacy of amlodipine and valsartan. CAs are presumed to be more effective in black than in white patients and, conversely, ARBs more effective in white than in black patients. ${ }^{30}$ There were only few black patients $(n=201)$ included in the population pooled from 2 controlled trials by Smith and colleagues, precluding a direct meaningful comparison of the blood pressure responses between the two ethnic groups. ${ }^{29}$ In both subgroups monotherapies were more effective than placebo, and combination therapies more effective than the corresponding component given alone.

Figure 4 illustrates the overall incidence of adverse events observed in patients allocated to amlodipine $(\mathrm{n}=460)$ and valsartan $(n=921)$, their combination $(n=1437)$ or placebo $(\mathrm{n}=337) .{ }^{29}$ Adverse events tended to be more frequent during
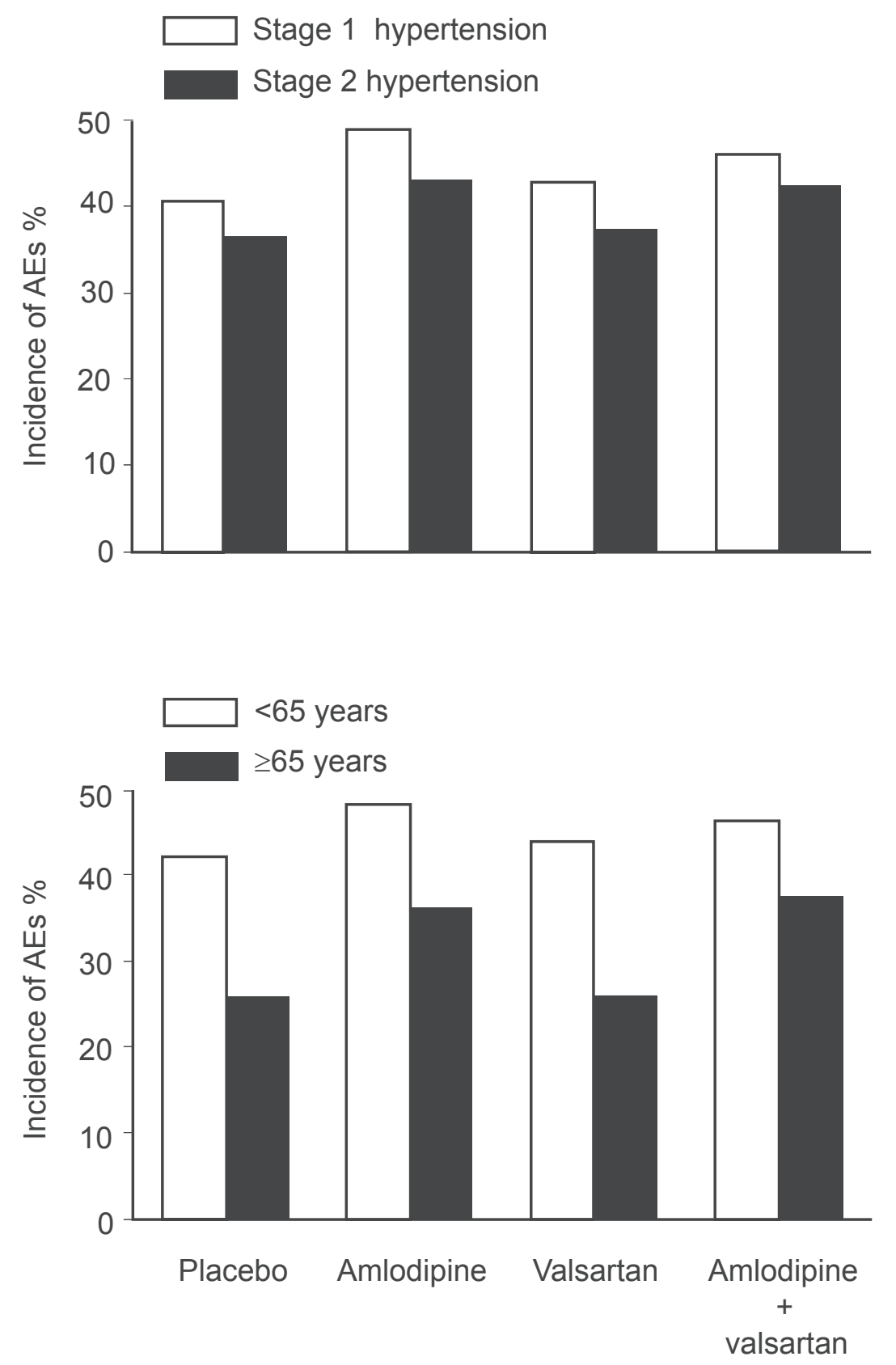

Figure 4 Overall incidence of adverse events (AEs) with amlodipine and valsartan, alone or in combination, in patients with stage I or stage 2 hypertension (upper panel), and in younger or older patients (lower panel). Adapted with permission from Smith et al.J Clin Hypertens (Greenwich). 2007;9:355-364.29 Copyright @ 2007 John Wiley \& Sons, Inc. 
amlodipine therapy in stage 1 than in stage 2 hypertension (upper panel). Valsartan given alone had a placebo-like tolerability regardless of the degree of blood pressure elevation at baseline. Amlodipine combined with valsartan exhibited in both hypertension stages a tolerability comparable to that of amlodipine administered as single agent. Notably, tolerability was substantially better in older than in younger patients (lower panel). This was true whether the patients received an active treatment or placebo. Valsartan and placebo were equally well tolerated. In both age groups, the incidence of adverse events was very similar when amlodipine was given alone or together with valsartan. Thus, the common belief that older patients do not tolerate antihypertensive therapy as well as younger patients was not confirmed using amlodipine and valsartan.

\section{Blockade of the renin-angiotensin system plus calcium entry blockade or diuretic therapy}

Dual therapy with a blocker of the renin-angiotensin system and a CA represents today an important therapeutic option, similar to that based on a blocker of the renin-angiotensin and a thiazide diuretic. ${ }^{6}$ It is worth mentioning here a study aimed to compare the efficacy and the tolerability of 2 combination regimens, one containing a $\mathrm{CA}$ (amlodipine) and an ARB (valsartan), and the other an ACE-I (lisinopril) and a diuretic (hydrochlorothiazide, HCTZ). ${ }^{31}$ The patients included in this trial had stage 2 hypertension, ie, patients in whom the use of a fixed-dose combination could be considered to initiate antihypertensive therapy. ${ }^{6,32}$ They were randomly allocated to receive for 6 weeks, according to a double-blind design, once-daily treatment with amlodipine $5 \mathrm{mg}$ and valsartan $160 \mathrm{mg}(\mathrm{n}=63)$, or lisinopril $10 \mathrm{mg}$ and HCTZ $12.5 \mathrm{mg}(\mathrm{n}=65)$. The doses of amlodipine and lisinopril were increased to $10 \mathrm{mg}$ and $20 \mathrm{mg}$, respectively, if diastolic blood pressure remained $\geq 90 \mathrm{mmHg}$ after the first 2 weeks of treatment. There was no significant difference in the blood pressure reductions achieved at the end of the 6-week follow-up between the two drug regimens (amlodipine-valsartan: 35.8/28.6 mmHg; lisinopril-HCTZ: $31.8 / 27.6 \mathrm{mmHg}$ ). Notably, both treatments were equally well tolerated.

The observations made by Poldermans and colleagues suggest that both types of combinations can be used indiscriminately in hypertensive patients, in terms both of antihypertensive efficacy and tolerability. One should keep in mind, however, that the trial was carried out according to a parallel-group design, which does not enable any conclusions to be drawn about individual responses. A given patient may normalize his/her blood pressure regardless of the content of the drug combination, or exclusively with one type of combination, or even be a non-responder to both combinations. The same is true for tolerability. Any drug combination might occasionally cause adverse events in a given patient. There is therefore still need for individualization of treatment when co-administering 2 antihypertensive agents with different mechanisms of action, the aim being to normalize blood pressure with no adverse impact on the patient's quality of life.

The main mechanism of action of ARBs and ACE-Is is related to the blocking effect of these drugs on the renin-angiotensin system. It is possible, however, that some accumulation of kinins occurs during ACE inhibition, which might contribute to the blood pressure-lowering effect of ACE-I. Combining an ARB and an ACE-I therefore appears attractive, not only to achieve maximal blockade of the renin-angiotensin system, but also to gain in antihypertensive efficacy via a bradykinin-induced release of $\mathrm{NO}$ from the endothelium. A study was performed in 64 patients with an ambulatory blood pressure not controlled by valsartan, $160 \mathrm{mg} /$ day, to compare the additional antihypertensive effects of the ACE-I benazepril, $20 \mathrm{mg} /$ day, the diuretic chlortalidone, $12.5 \mathrm{mg} /$ day, or amlodipine, $5 \mathrm{mg} /$ day. $^{33}$ These three types of agents were administered on top of valsartan, $160 \mathrm{mg} /$ day, for 5 -week periods. Combining valsartan and benazepril led to a significant additional decrease in 24-h ambulatory blood pressure $(-8.6 /-6.3 \mathrm{mmHg})$. This was, however, significantly less than the comparative 24-h ambulatory blood pressure reduction obtained by co-administering valsartan and amlodipine $(-15.2 /-9.9 \mathrm{mmHg})$ or valsartan and chlortalidone $(-13.5 / 9.5 \mathrm{mmHg})$.

Another key issue is related to the potential impact of various combinations on cardiovascular and renal outcomes. An ACE-I (benazepril, 20-40 mg)-CA (amlodipine, 5-10 mg) combination ( $\mathrm{n}=5713$ ) and a ACE-I (benazepril, 20-40 mg)diuretic (HCTZ, 12.5-25 mg) combination $(\mathrm{n}=5733)$ have been directly compared recently in patients aged $>55$ years with either a systolic blood pressure $\geq 160 \mathrm{mmHg}$ or currently on antihypertensive therapy. An additional inclusion criterion was the existence of cardiovascular, renal disease or other target organ damage. ${ }^{34}$ In this trial, known as the ACCOMPLISH Study (Avoiding Cardiovascular events through COMbination therapy in Patients Living with Systolic Hypertension), the aim was to lower blood pressure below $140 \mathrm{mmHg}$ in most patients, and below 130/80 mmHg 
in patients with diabetes or renal insufficiency. To this end the doses of the study drugs could be titrated within the first 3 months, and additional antihypertensive agents added thereafter if required. The trial was stopped prematurely in March 2008 because of a significant lower cardiovascular mortality and morbidity in the benazepril-amlodipine group compared with the benazepril-HCTZ group (risk ratio [95\% $\mathrm{CI}]=0.80[0.72-0.90])$. The preliminary results of the ACCOMPLISH Study have been presented at the Annual Scientific Sessions of the ACC, on March 31, 2008 by Professor Kenneth Jamerson in Chicago, USA. The blood pressure control rate at completion of the trial was high and comparable in the two groups $(81.7 \%$ in the benazepril-amlodipine group and $78.5 \%$ in the benazepril-HCTZ group). The final results have been released very recently. ${ }^{35}$ The benazepril-amlodipine drug regimen was found to reduce significantly the relative risk of developing events such as fatal and non-fatal myocardial infarction ( $-22 \%)$ and coronary revascularization $(-14 \%)$, suggesting that this type of combination is particularly effective in hypertensive patients for the prevention of complications caused by myocardial ischemia. These data are of great interest as the ACCOMPLISH Study is the first outcome trial to compare fixed-dose combinations. The subgroup analysis will tell us if some patients are more likely to benefit from an ACE-I-CA combination than from an ACE-I-HCTZ combination.

\section{Perspectives}

The increasing popularity of fixed-dose combinations in the treatment of hypertension is justified. Co-administering two antihypertensive agents with complementary mechanisms of action enables a better blood pressure control, but not at the expense of a deterioration in tolerability. Also, the simplification of treatment afforded by single tablets containing 2 active drugs facilitates long-term compliance with therapy, which is expected to affect positively cardiovascular and renal outcome. The fixed-dose combination based on a dihydropyridine $\mathrm{CA}$ and an ARB is a good example of an effective and well-tolerated fixed-dose combination. Such agents have no adverse metabolic impact and have been shown to have protective effects in large morbidity-mortality trials. $^{3-6}$ It appears therefore more and more appealing to block at the same time the renin-angiotensin system and calcium channels in the vasculature.

\section{Disclosures}

The authors have received consulting and lecturing fees from different pharmaceutical companies, including Novartis.

\section{References}

1. He J, Whelton PK. Epidemiology and prevention of hypertension. Med Clin North Am. 1997;81:1077-1097.

2. Ezzati M, Lopez AD, Rodgers A, Vander Hoorn S, Murray CJ. Selected major risk factors and global and regional burden of disease. Lancet. 2002;360:1347-1360.

3. Turnbull F, Neal B, Algert C, et al. Effects of different blood pressure-lowering regimens on major cardiovascular events in individuals with and without diabetes mellitus: results of prospectively designed overviews of randomized trials. Arch Int Med. 2005;165:1410-1419.

4. Staessen JA, Li Y, Thijs L, Wang JG. Blood pressure reduction and cardiovascular prevention: an update including the 2003-2004 secondary prevention trials. Hypertens Res. 2005;28:385-407.

5. Williams B. Recent hypertension trials: implications and controversies. J Am Coll Cardiol. 2005;45:813-827.

6. Mancia G, De Backer G, Dominiczak A, et al. 2007 Guidelines for the Management of Arterial Hypertension: The Task Force for the Management of Arterial Hypertension of the European Society of Hypertension (ESH) and of the European Society of Cardiology (ESC). J Hypertens. 2007;25:1105-1187.

7. Dickerson CJE, Hingorani AD, Ashby MJ, Palmer CR, Brown MJ. Optimisation of antihypertensive treatment by crossover rotation of four major classes. Lancet. 1999;353:2008-2013.

8. Law MR, Wald NJ, Morris JK, Jordan RE. Value of low dose combination treatment with blood pressure lowering drugs: analysis of 354 randomised trials. BMJ. 2003;326:1427-1431.

9. Dezii CM. A retrospective study of persistence with single-pill combination therapy vs concurrent two-pill therapy in patients with hypertension. Manag Care. 2000;9(Suppl):2-6.

10. Plosker GL, Robinson DM. Amlodipine/Valsartan: fixed-dose combination in hypertension. Drugs. 2008;68:373-381.

11. Abernethy DR, Schwartz JB. Calcium-antagonist drugs. N Engl J Med. 1999;341:1447-1457.

12. Luscher TF, Cosentino F. The classification of calcium antagonists and their selection in the treatment of hypertension. Drugs. 1998;55:509-517.

13. Meredith PA, Elliott HL. Clinical pharmacokinetics of amlodipine. Clin Pharmacokinet. 1992;22:22-31.

14. Grossman E, Messerli FH. Effect of calcium antagonists on plasma norepinephrine levels, heart rate, and blood pressure. Am J Cardiol. 1997;80:1453-1458.

15. Beevers DG, Sleight P. Short acting dihydropyridine (vasodilating) calcium channel blockers for hypertension: is there a risk? BMJ. 1996;312:1143-1145.

16. Binggeli C, Corti R, Sudano I, Luscher TF, Noll G. Noll G. Effects of chronic calcium channel blockade on sympathetic nerve activity in hypertension. Hypertension. 2002;39:892-896.

17. Markham A, Goa KL. Valsartan. A review of its pharmacology and therapeutic use in essential hypertension. Drugs. 1997;54:299-311.

18. Thurmann PA. Valsartan: a novel angiotensin type 1 receptor antagonist. Exp Opin Pharmacother. 2000;1:337-350.

19. Niarchos AP, Pickering TG, Morganti A, Laragh JH. Plasma catecholamines and cardiovascular responses during converting enzyme inhibition in normotensive and hypertensive man. Clin Exp Hypertens. 1982;4:761-789.

20. Seljeflot I, Moan A, Kjeldsen S, Sandvik E, Arnesen H. Effect of angiotensin II receptor blockade on fibrinolysis during acute hyperinsulinemia in patients with essential hypertension. Hypertension. 1996;27:1299-1304.

21. Julius S, Kjeldsen SE, Weber M, et al. Outcomes in hypertensive patients at high cardiovascular risk treated with regimens based on valsartan or amlodipine: the VALUE randomised trial. Lancet. 2004;363:2022-2031.

22. Messerli FH. Vasodilatory edema: a common side effect of antihypertensive therapy. Curr Cardiol Rep. 2002;4:479-482.

23. Sever P. New hypertension guidelines from the National Institute for Health and Clinical Excellence and the British Hypertension Society. J Renin Angiotensin Aldosterone Syst. 2006;7:61-63. 
24. Textor SC. Renovascular hypertension in 2007: where are we now? Curr Cardiol Rep. 2007;9:453-461.

25. Gennari C, Nami R, Pavese G, Gragnani S, Bianchini C, Buracchi P. Calcium-channel blockade (nitrendipine) in combination with ACE inhibition (captopril) in the treatment of mild to moderate hypertension. Cardiovasc Drugs Ther. 1989;3 Suppl 1:319-325.

26. Luft FC, Aronoff GR, Sloan RS, Fineberg NS, Weinberger MH. Calcium channel blockade with nitrendipine. Effects on sodium homeostasis, the renin-angiotensin system, and the sympathetic nervous system in humans. Hypertension. 1985; 7:438-442.

27. Philipp T, Smith TR, Glazer R, et al. Two multicenter, 8-week, randomized, double-blind, placebo-controlled, parallel-group studies evaluating the efficacy and tolerability of amlodipine and valsartan in combination and as monotherapy in adult patients with mild to moderate essential hypertension. Clin Ther. 2007;29:563-580.

28. Fogari R, Zoppi A, Derosa G, et al. Effect of valsartan addition to amlodipine on ankle oedema and subcutaneous tissue pressure in hypertensive patients. J Hum Hypertens. 2007;21:220-224.

29. Smith TR, Philipp T, Vaisse B, et al. Amlodipine and valsartan combined and as monotherapy in stage 2, elderly, and black hypertensive patients: subgroup analyses of 2 randomized, placebo-controlled studies. J Clin Hypertens (Greenwhich). 2007;9:355-364.

30. Materson BJ, Reda DJ, Cushman WC, et al. Single-drug therapy for hypertension in men. A comparison of six antihypertensive agents with placebo. The Department of Veterans Affairs Cooperative Study Group on Antihypertensive Agents. N Engl J Med. 1993;328:914-921.
31. Poldermans D, Glazes R, Kargiannis S, et al. Tolerability and blood pressure-lowering efficacy of the combination of amlodipine plus valsartan compared with lisinopril plus hydrochlorothiazide in adult patients with stage 2 hypertension. Clin Ther. 2007;29:279-289.

32. Chobanian AV, Bakris GL, Black HR, et al. Seventh report of the Joint National Committee on Prevention, Detection, Evaluation, and Treatment of High Blood Pressure. Hypertension. 2003;42:1206-1252.

33. Stergiou GS, Makris T, Papavasiliou M, Efstathiou S, Manolis A. Comparison of antihypertensive effects of an angiotensin-converting enzyme inhibitor, a calcium antagonist and a diuretic in patients with hypertension not controlled by angiotensin receptor blocker monotherapy. J Hypertens. 2005;23:883-889.

34. Jamerson KA, Bakris GL, Wun CC, et al. Rationale and design of the avoiding cardiovascular events through combination therapy in patients living with systolic hypertension (ACCOMPLISH) trial: the first randomized controlled trial to compare the clinical outcome effects of first-line combination therapies in hypertension. Am J Hypertens. 2004;17:793-801.

35. Jamerson K, Weber MA, Bakris GL, et al. Benazepril plus amlodipine or hydrochlorothiazide for hypertension in high-risk patients. $N$ Engl $J$ Med. 2008;359:2417-2428. 